\title{
Genetische Feintypisierung
}

\section{SNP-Typisierung des Buruli-Ulkus-Erregers}

KATHARINA RÖLTGEN, GERD PLUSCHKE

SCHWEIZERISCHES TROPEN- UND PUBLIC HEALTH-INSTITUT, MOLEKULARE

IMMUNOLOGIE, BASEL, SCHWEIZ

\section{Mithilfe vergleichender Genomanalysen konnten wir eine Feintypisie- rungsmethode für Mycobacterium ulcerans, dem genetisch monomor- phen Erreger des Buruli-Ulkus, entwickeln.}

Based on comparative genomic analyses we have developed a fine-typing method for Mycobacterium ulcerans, the genetically monomorphic causative agent of Buruli ulcer.

Das Buruli-Ulkus ist eine von Mycobacterium ulcerans hervorgerufene Hauterkrankung, die vor allem in Feuchtgebieten Westafrikas, aber auch in Teilen Australiens, Asiens und Lateinamerikas auftritt.
M. ulcerans gehört zur gleichen Familie wie die Tuberkel- und Leprabazillen. Im Gegensatz zu diesen produziert $M$. ulcerans ein Makrolid-Toxin, das durch lokale Gewebezerstörung die für das Buruli-Ulkus typischen
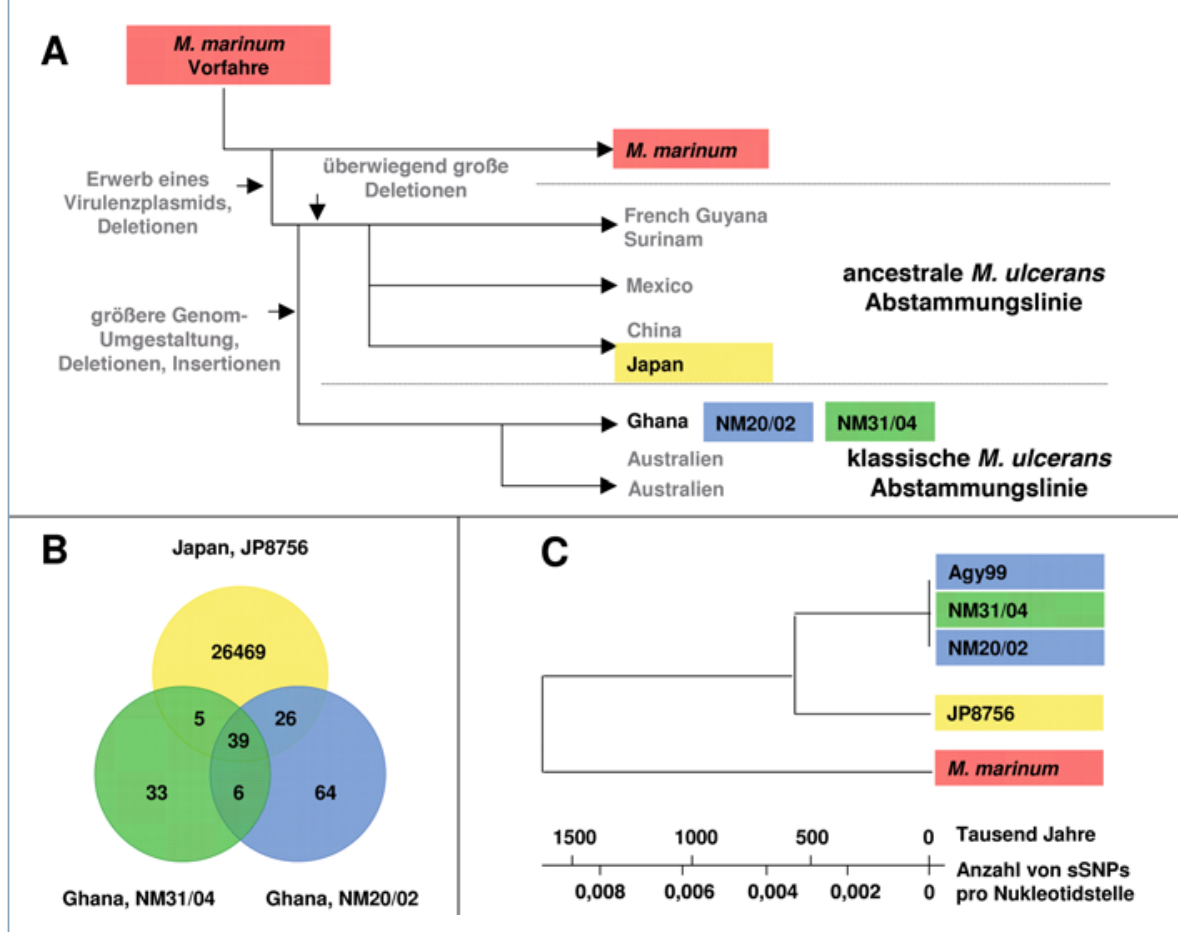

A Abb. 1: Genetische Typisierung von Mycobacterium ulcerans. A, Die Analyse von großen Sequenzpolymorphismen innerhalb von $M$. ulcerans-Isolaten weltweiten Ursprungs ermöglichte eine Unterscheidung von fünf kontinentalen Haplotypen und zwei Abstammungslinien. B, Vergleichende Genomanalysen erlaubten die Detektion von SNPs. C, Abschätzung der Entstehungszeit genetischer M. ulcerans-Varianten basierend auf sSNPs.

chronischen, fortschreitenden Geschwüre verursacht. Unbehandelt kommt es im Verlauf der M. ulcerans-Infektion zu teilweise großflächigen Hautläsionen, die nach einigen Monaten oder Jahren zu schweren Deformationen bis hin zum Verlust von Körperteilen führen können. Derzeit empfiehlt die Weltgesundheitsorganisation (WHO) eine achtwöchige Antibiotikatherapie mit Rifampicin und Streptomycin. Obwohl das Buruli-Ulkus die dritthäufigste mykobakterielle Erkrankung nach Tuberkulose und Lepra ist und die WHO 1998 eine „Globale Buruli-Ulkus-Initiative" initiierte, ist die Krankheit wissenschaftlich noch unzureichend erforscht. Es gibt weder einen Impfstoff noch eine einfache diagnostische Methode zur Früherkennung.

Bis heute sind die Übertragungswege des Erregers nicht vollständig geklärt. Es wird jedoch angenommen, dass $M$. ulcerans durch Kontakt mit einem noch nicht identifizierten, Wasser-assoziierten Umweltreservoir über Hautverletzungen aufgenommen wird und dass eine direkte Weitergabe von Mensch zu Mensch unwahrscheinlich ist. Für ein besseres Verständnis der Epidemiologie des Buruli-Ulkus werden Methoden benötigt, die eine Unterscheidung von genetischen Varianten des Erregers ermöglichen. Mithilfe einer hochauflösenden Typisierungsmethode könnte die räumliche und zeitliche Ausbreitung genetischer Varianten erforscht werden, um Umweltreservoire und vermeidbare Infektionsrisiken zu identifizieren.

\section{Typisierung genetisch monomorpher Organismen}

Wie auch einige andere hochvirulente bakterielle Erreger weist M. ulcerans außergewöhnlich wenig genetische Diversität auf. Die einfachste Erklärung für die Entstehung von derart genetisch „monomorphen“ Pathogenen ist, dass sie die Nachkommen eines Klons sind, der vor relativ kurzer Zeit eine entscheidende und einmalige genetische Veränderung erfahren hat. Bei M. ulcerans steht ein genetischer „Flaschenhals-Effekt" vermutlich 


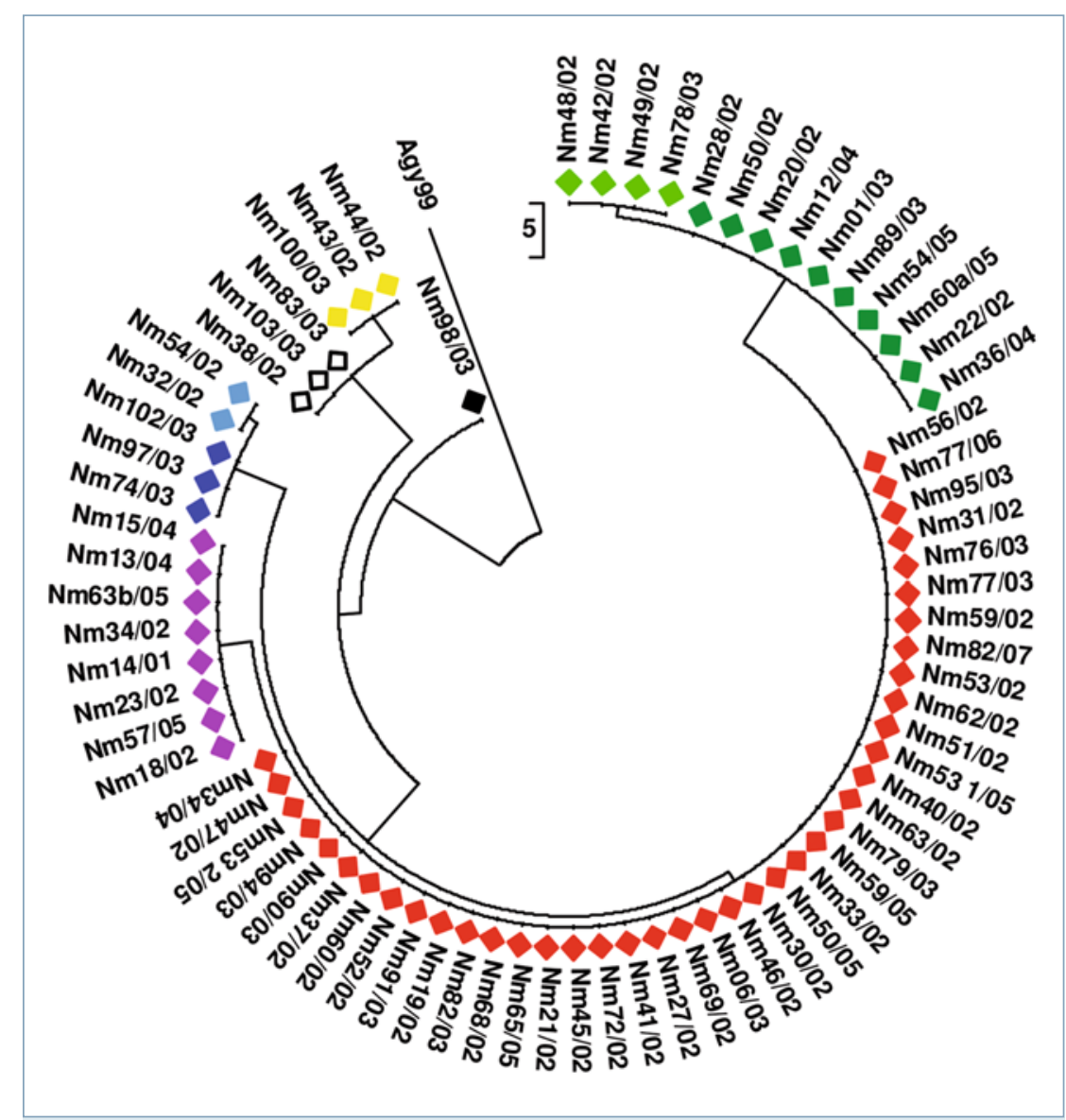

$\Delta$ Abb. 2: Verwandtschaftsbaum. 75 Mycobacterium ulcerans-Stämme, die von Patienten aus dem Densu-Flussgebiet in Ghana isoliert worden waren, konnten mittels genetischer Feintypisierung in zehn M. ulcerans-Haplotypen eingeteilt werden (Skala: Anzahl der detektierten SNPs).

mit dem Erwerb eines Virulenzplasmids in Zusammenhang, durch den sich der Erreger vor evolutionär relativ kurzer Zeit aus dem Fischpathogen Mycobacterium marinum entwickelt hat [1]. Aufgrund der geringen DNA-Sequenzunterschiede zwischen Varianten eines monomorphen Pathogens ist es im Vergleich zu genetisch vielfältigen Mikroorganismen schwierig, Feintypisierungsmethoden zu finden, die Rückschlüsse auf Epidemiologie und Evolutionsgeschichte des Erregers zulassen. Folglich ist es nicht verwunderlich, dass das Auflösungsvermögen konventioneller genetischer Typisierungsmethoden bei M. ulcerans für mikroepidemiologische Analysen nicht hinreichend ist. Genomanalysen mit anderen genetisch monomorphen Pathogenen, wie Mycobacterium tuberculosis, haben gezeigt, dass Sequenzpolymorphismen in Form von Inversionen, Insertionen oder Deletionen (large sequence polymorphisms, LSPs) oder Einzelnukleotid-Polymorphismen (single nucleotide polymorphisms, SNPs) geeignete gene- tische Marker für phylogenetische Analysen darstellen [2]. Besonders SNP-Analysen sind bei hochklonalen Pathogenen sehr informativ, da sich daraus retrospektiv eine klare, binär aufteilende Phylogenie ableiten lässt.

Bei M. ulcerans konnten mittels LSPAnalysen zwar weltweit mehrere kontinentale Haplotypen und zwei Abstammungslinien, die anzestrale (Stämme aus Asien, Südamerika und Mexiko) und die klassische Linie (Stämme aus Afrika, Australien und Südostasien) unterschieden werden (Abb. 1A, [3]). Jedoch haben wir bei mikroepidemiologischen Studien innerhalb von lokalen M. ulcerans-Populationen keine LSP-Varianten gefunden. Daher verblieb die Identifizierung von SNPs als einzige Option für eine genetische Feintypisierung.

\section{Vergleichende Genomanalyse von Mycobacterium ulcerans}

Die im Jahr 2007 veröffentlichte Genomsequenz eines M. ulcerans-Stamms (Agy99 
aus Ghana [4]) lieferte die Voraussetzung für vergleichende Genomanalysen bei diesem Erreger. Vergleiche mit dem Genom von M. marinum (6,6 Megabasen) zeigten, dass das M. ulcerans-Genom eine geringere Größe (5,6 Megabasen) aufweist. Es wird vermutet, dass diese ca. 15-prozentige Genomreduktion mit der Adaptation an eine stabile ökologische Nische zusammenhängt. Um eine SNP-Typisierungsmethode für die zur klassischen Linie gehörenden ghanaischen M. ulcerans-Stämme zu entwickeln, haben wir die Genome von zwei weiteren ghanaischen M. ulcerans-Isolaten mittels der Roche-454GS-FLX- bzw. der Illumina-Solexa-Sequenzierungstechnologie sequenziert. Wie Agy99 wurde der eine Stamm (NM20/02) von einem Patienten aus dem Densu-Flussgebiet im Süden Ghanas isoliert, während der zweite Stamm (NM31/04) von einem Patienten aus einem anderen Buruli-Ulkus-endemischen Gebiet im Osten Ghanas stammte. Zudem wurde ein japanischer Patientenstamm (JP8756) als Vertreter der weniger virulenten anzestralen Abstammungslinie für eine Illu-
mina-Solexa-Sequenzierung ausgewählt. Die generierten DNA-Sequenzen wurden mithilfe von speziellen Kartierungsprogrammen (gsMapper, MAQ) auf die Agy99-Referenzsequenz abgebildet. Beide Sequenzierungstechnologien lieferten hierfür geeignete Datensätze. Der Genomvergleich mit dem Stamm Agy99 ergab 135 SNPs für NM20/02, 83 SNPs für NM31/04 und 26.564 SNPs für JP8756 (Abb. 1B, [5]). Bei dem japanischen Isolat JP8756 führte die Mehrzahl der SNPs (63 Prozent) in codierenden Regionen zu keinem Aminosäureaustausch (synonyme SNPs, sSNPs). Hingegen dominierten bei den sequenzierten ghanaischen Stämmen SNPs, die zu einem Aminosäureaustausch in der Sequenz führten (nicht-synonyme SNPs, nsSNPs), jedoch vermutlich keine funktionelle Relevanz haben. Synonyme SNPs stellen in klonalen Populationen eine Art molekulare Uhr dar, mit der ihre Entstehungszeit errechnet werden kann. Mithilfe der GenomSequenzierungsdaten konnte daher die vor relativ kurzer Zeit erfolgte Divergenz von M. marinum und M. ulcerans von einem

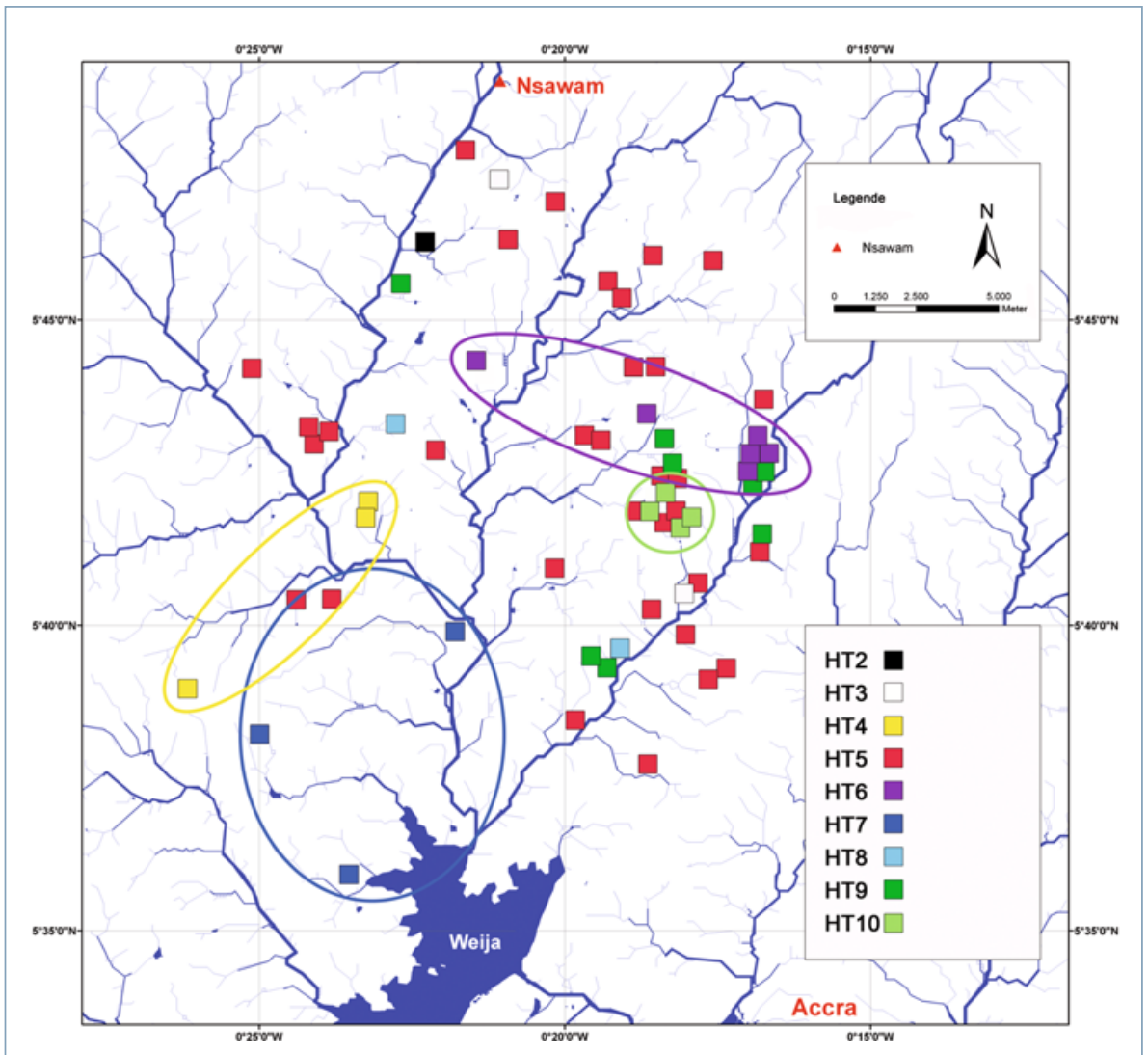

A Abb. 3: Geografische Verteilung von Mycobacterium ulcerans-Haplotypen im Densu-Flussgebiet. Die Haplotypen 2, 3, 4, 6, 7, 8, 9 und 10 sind ungleichmäßig verteilt, während Haplotyp 5 überall vorkommt. Haplotyp 1 (Agy99) ist nicht eingetragen. Die Gewässer wurden mit optischen (Landsat ETM) und Radar-Daten (TerraSAR-X) detektiert. gemeinsamen Vorfahren auf einen Zeitpunkt vor etwa 1,3 Millionen Jahren abgeschätzt werden. Entsprechend divergierten die klassische und die anzestrale Linie von M. ulcerans vor etwa 400.000 Jahren. Die Divergenz der ghanaischen Stämme liegt vergleichsweise sehr kurz (unter 3.000 Jahre) zurück (Abb. 1C).

Interessant ist, dass 100 nicht-synonyme SNPs in JP8756 ein verfrühtes Stoppcodon verursachten, während dies bei den in den ghanaischen Stämmen identifizierten SNPs nie vorkam. Da wir innerhalb einer Sammlung ghanaischer $M$. ulcerans-Isolate auch keine LSPs finden konnten, vermuten wir, dass es bei der klassischen Linie von M. ulcerans zu einer Stabilisierung des Genoms gekommen ist und keine weitere rasche Reduktion des Genoms mehr erfolgt.

\section{SNP-Typisierung nah verwandter Mycobacterium ulcerans-Isolate}

Mithilfe der bei den ghanaischen M. ulceransStämmen identifizierten SNPs haben wir eine auf der Real-Time-Polymerasekettenreaktion (RT-PCR) basierende hochauflösende Typisierungsmethode entwickelt. Insgesamt 65 einzelne RT-PCR-SNP-Assays ermöglichten es, M. ulcerans-Stämme aus Ghana mit mittlerer Durchsatzgeschwindigkeit zu typisieren. In Zusammenarbeit mit dem Noguchi Memorial Institute for Medical Research in Ghana haben wir 75 Stämme von BuruliUlkus-Patienten aus dem Densu-Flussgebiet von Ghana isoliert und typisiert. Hierbei konnten bei den zwischen 2001 und 2007 gewonnenen Isolaten fünf Haplotypen (HT2-6) identifiziert werden, die sich vom Haplotyp des Referenzstamms Agy99 (HT1) unterschieden. In einem nächsten Schritt konnte durch Sequenzierung der Genome ausgewählter weiterer Stämme eine noch weitergehende Feintypisierung der 75 Isolate aus dem Densu-Flussgebiet erreicht werden. Jeweils ein Vertreter der Haplotypen 3 bis 6 wurde zur Identifizierung weiterer SNPs mit der Illumina-Technologie sequenziert. Beim Genomvergleich der vier Haplotypenvertreter untereinander und mit dem Referenzgenom Agy99 wurden insgesamt 66 neue SNPs gefunden. Für 24 von ihnen konnten wir zusätzliche RTPCR-SNP-Assays entwerfen, die gemeinsam mit den schon etablierten 65 Assays eine Unterteilung der 75 aus dem Densu-Flussgebiet kommenden Stämme in insgesamt zehn Haplotypen (HT1-10) erlaubte (Abb. 2, [6]). Die detektierten Haplotypen 1 bis 10 scheinen Nachkommen eines gemeinsamen Vor- 
fahren mit dem Gründer-Haplotyp $5 \mathrm{zu}$ sein, der im gesamten Densu-Flussgebiet zu finden war (Abb. 3). Die Verbreitung der meisten anderen Haplotypen war hingegen jeweils auf einen bestimmten Teil des Buruli-Ulkus-endemischen Gebiets begrenzt. Dies spricht für eine fokale Übertragung von neu entstandenen genetischen Varianten des dominierenden Klons und damit gegen eine Übertragung durch direkten Kontakt mit verunreinigtem Flusswasser. Auch eine Übertragung durch Insektenspezies, die über weite Distanzen fliegen können, ist unwahrscheinlich. Unsere Ergebnisse weisen vielmehr darauf hin, dass chronische Läsionen bei Tieren und/oder Menschen ein M. ulceransReservoir darstellen. Hierfür spricht auch der Verlust von immundominanten Antigenen bei der Genomreduktion von M. ulcerans [7]. Während in einem Buruli-Ulkus-endemischen Gebiet Australiens zahlreiche mit $M$. ulcerans infizierte Possums gefunden worden sind [8], konnte in den endemischen Gebieten Afrikas noch kein solches Tierreservoir identifiziert werden. Da das Buruli-Ulkus primär in Feuchtgebieten vorkommt, vermuten wir, dass M. ulcerans von chronischen Wunden aus in eine mit stehendem Wasser assoziierte Umweltnische gelangt. Nicht auszuschlieBen ist, dass es sich hierbei um Insekten mit geringer Mobilität handelt, die vorwiegend in der Nähe lebende Tiere und/oder Menschen infizieren. Weiterführende prospektive mikroepidemiologische Studien sind erforderlich, um das Rätsel um die fokale Übertragung von M. ulcerans zu lösen. Eine Identifizierung von vermeidbaren Risikofaktoren für eine
Infektion könnte wesentlich zur Kontrolle der Krankheit beitragen.

\section{Danksagung}

Ein Teil dieser Arbeiten wurde von der Stop Buruli-Initiative gefördert, die von der UBS-Optimus-Foundation unterstützt wird.

\section{Literatur}

[1] Stinear TP, Jenkin GA, Johnson PDR et al. (2000) Comparative genetic analysis of Mycobacterium ulcerans reveals evidence of recent divergence. J Bacteriol 182:6322-6330

[2] Gagneux S, Small PM (2007) Global phylogeography of Mycobacterium tuberculosis and implications for tuberculosis product development. Lancet Infect Dis 7:328-337 [3] Käser M, Rondini S, Naegeli M et al. (2007) Evolution of two distinct phylogenetic lineages of the emerging human pathogen Mycobacterium ulcerans. BMC Evol Biol 7:177

[4] Stinear TP, Seemann T, Pidot S et al. (2007) Reductive evolution and niche adaptation inferred from the genome of Mycobacterium ulcerans, the causative agent of Buruli ulcer. Genome Res 17:192-200

[5] Oi W, Käser M, Röltgen K et al. (2009) Genomic diversity and evolution of Mycobacterium ulcerans revealed by next-generation sequencing. PloS Pathog 5:e1000580 [6] Röltgen K, Qi W, Ruf M et al. (2010) Single nucleotide polymorphism typing of Mycobacterium ulcerans reveals focal transmission of buruli ulcer in a highly endemic region of Ghana. PloS Negl Trop Dis 4:e751

[7] Huber CA, Ruf M, Pluschke G et al. (2008) Independent loss of immunogenic proteins in

Mycobacterium ulcerans suggests immune evasion. Clin Vaccine Immunol 15:598-606

[8] Fyfe JA, Lavender CJ, Handasyde KA et al. (2010) A major role for mammals in the ecology of Mycobacterium ulcerans. PloS Negl Trop Dis 4:e791

\section{Korrespondenzadresse:}

Prof. Dr. Gerd Pluschke

Schweizerisches Tropen- und Public HealthInstitut

Socinstraße 57

$\mathrm{CH}-4002$ Basel

Tel.: +41-(0)61-284-8235

Fax: +41-(0)61-284-8101

gerd.pluschke@unibas.ch

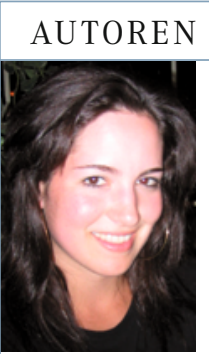

Katharina Röltgen

Jahrgang 1984. 2005-2007 Molekularbiologie-Bachelorstudium an der Universität Mainz. 2007-2008 Infektionsbiologie-Masterstudium am Schweizerischen Tropenund Public Health-Institut, Universität Basel. Seit 2009 Doktorarbeit in der Arbeitsgruppe von Prof. Dr. Pluschke, Schweizerisches Tropen- und Public Health-Institut, Universität Basel.

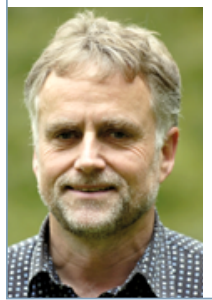

Gerd Pluschke

Jahrgang 1952. Biochemiestudium in Tübingen und München. 1979 Promotion. Postdoc und Wissenschaftlicher Mitarbeiter am Max-Planck-Institut für Molekulare Genetik, Berlin, dem Basler Institut für Immunologie und der Ciba Pharmaforschung. Seit 1995 Leiter der Einheit Molekulare Immunologie und seit 2008 des Departments Medizinische Parasitologie und Infektionsbiologie des Schweizerischen Tropen- und Public Health-Instituts in Basel. 